LUNG CANCER

\title{
Effect of comorbidity on the treatment and prognosis of elderly patients with non-small cell lung cancer
}

\author{
M L G Janssen-Heijnen, S Smulders, V E P P Lemmens, F W J M Smeenk, H J A A van Geffen, \\ J W W Coebergh
}

Thorax 2004;59:602-607. doi: 10.1136/thx.2003.018044

See end of article for authors' affiliations auther affiliations

Correspondence to: $\operatorname{Dr} M$ L G Janssen-Heijnen, Eindhoven Cancer Registry, Comprehensive Cancer Center South (IKZ), $\mathrm{PO}$ Box 231, $5600 \mathrm{AE}$ Eindhoven, The Netherlands; research@ikz.nl

Received 4 November 2003 Accepted 3 March 2004

\begin{abstract}
Background: With the rising mean age, more patients will be diagnosed with one or more other serious diseases at the time of lung cancer diagnosis. Little is known about the best way to treat elderly patients with comorbidity or the outcome of treatment. This study was undertaken to evaluate the independent effects of age and comorbidity on treatment and prognosis in patients with non-small cell lung cancer (NSCLC).

Methods: All patients with NSCLC diagnosed between 1995 and 1999 in the southern part of the Netherlands ( $\mathrm{n}=4072$ ) were included.

Results: The proportion of patients with localised NSCLC who underwent surgery was $92 \%$ in patients younger than 60 years and $9 \%$ in those aged 80 years or older. In patients aged 60-79 years this proportion also decreased with comorbidity. In patients with non-localised NSCLC the proportion receiving chemotherapy was considerably higher for those aged less than 60 years $(24 \%)$ than in those aged 80 or older (2\%). The number of comorbid conditions had no significant influence on the treatment chosen for patients with non-localised disease. Multivariable survival analyses showed that age, tumour size, and treatment were independent prognostic factors for patients with localised disease, and stage of disease and treatment for those with non-localised disease. Comorbidity had no independent prognostic effect.

Conclusions: It is questionable whether the less aggressive treatment of elderly patients with NSCLC is justified.
\end{abstract}

ung cancer occurs mainly in the elderly. Because of a demographic shift towards an older population and -improved survival of patients with cardiovascular diseases, more elderly people are at risk of developing lung cancer. The proportion of patients aged 70 or older has increased from $26 \%$ in 1970 to $43 \%$ in $2000 .{ }^{1}$ With the rising mean age, more patients will be diagnosed with one or more other serious diseases at the time of lung cancer diagnosis (comorbidity). ${ }^{2}$ Comorbidity may lead to complications during or after surgical and/or systemic treatment. ${ }^{3-6}$ The clinical management of lung cancer is therefore becoming increasingly complex. Furthermore, these patients are often excluded from clinical trials. This means that little is known about the best way to treat elderly patients with comorbidity and about the outcome of treatment such as complications and survival.

Population based cancer registries are able to facilitate studies of these patients if they collect data directly from medical records. The Eindhoven Cancer Registry, at the request of clinicians, has been collecting data on clinically relevant concomitant diseases for all cancer patients diagnosed since 1993 in the southern part of the Netherlands. We report here on the influence of age, stage of disease, and comorbidity on the choice of treatment and survival of patients with non-small cell lung cancer (NSCLC).

\section{METHODS}

The Eindhoven Cancer Registry records data on all patients newly diagnosed with cancer in the southern part of the Netherlands, an area with about two million inhabitants. Since 1993 serious comorbidity with an impact on prognostis has also been recorded for all patients according to a slightly adapted version of the index developed by Charlson et $^{7 l^{7}}$ (box
1). The data were extracted from previous admissions, letters from and to other specialists, medical history, and preoperative screening. Comorbidity was defined as diseases that were present at the time of the cancer diagnosis.

Patients with NSCLC diagnosed between 1995 and 1999 $(\mathrm{n}=4076)$ were included. Those with cancer diagnosed at post mortem examination $(\mathrm{n}=138)$ were excluded.

Clinical tumour staging was performed according to the Tumour-Node-Metastasis (TNM) system of the Union Internationale Contre le Cancer version $4 .^{8}$ Tumours were classified as localised (stages I and II) and non-localised (stages III and IV). Non-small cell lung tumours were classified as squamous cell carcinoma, adenocarcinomas, and large cell undifferentiated carcinoma according to the WHO classification. ${ }^{9}$

Treatment for localised disease was classified as surgery (with or without adjuvant radiotherapy), radiotherapy alone, and "other or none". Treatment was only classified as surgery when the tumour was resected during surgical intervention; diagnostic surgery was not included. For patients with nonlocalised disease treatment was classified as surgery (with or without radiotherapy), radiotherapy alone, chemotherapy (with or without radiotherapy) and "other or none".

Data on vital status were available up to 1 April 2002. In addition to passive follow up via the hospitals, information was also obtained from the municipal registries in the area of the Eindhoven Cancer Registry and the Central Bureau for Genealogy, an institution that collects data on all deceased Dutch citizens via the civil municipal registries. In this way, information on patients who moved outside the registry area was also obtained. Patients who died outside the Netherlands were lost to follow up. The estimated proportion of these patients was $0.2 \%$. Of 4076 patients with NSCLC, 637 (16\%) were still alive and 3439 (84\%) were dead at the end of the study. 
Box 1 Classification of comorbidity according to an adapted version of the list of Charlson et $\mathrm{al}^{7}$

- Chronic obstructive pulmonary diseases (COPD);

- Cardiovascular diseases (e.g. myocardial infarction, cardiac decompensation, angina pectoris, peripheral arterial disease, intermittent claudication, abdominal aneurysm);

- Cerebrovascular diseases (cerebrovascular accident, hemiplegia);

- Other malignancies (except basal cell skin carcinoma);

- Hypertension (medically treated);

- Diabetes mellitus;

- Other:

- soft tissue diseases (e.g. Besnier Boeck disease, Wegener's disease, systemic lupus erythematosus);

- rheumatoid arthritis (only severe);

- kidney diseases (chronic glomerulonephritis, chronic pyelonephritis);

- bowel diseases (Crohn's disease, ulcerative colitis);

- liver diseases (cirrhosis, hepatitis);

- dementia;

- chronic infections

\section{Analysis of data}

Overall survival rates were computed (3 year for patients with localised disease and 1 year for patients with nonlocalised disease). Survival time was defined as the time from diagnosis to death or the end of the study (if the patient was still alive on 1 April 2002). The log rank test was performed to evaluate significant differences between survival curves in univariate analyses. For evaluation of the independent effects of the prognostic factors a multivariable Cox regression model was built. The models were stratified according to stage. This was done because both the guidelines for treatment of localised NSCLC and the survival rates are clearly different from those for non-localised NSCLC. Since interaction terms with age were not statistically significant, multivariable analyses were not stratified according to age. The independent prognostic effects of age, sex, stage of disease, and comorbidity were first estimated using a model without treatment. Treatment was then included in the model to investigate whether the prognostic effects of age and comorbidity could be fully explained by less aggressive treatment. With respect to comorbidity, the prognostic effects of both the number of comorbid conditions and the specific diseases/combinations of diseases were evaluated.

Survival generally decreases with age and the prevalence of comorbidity increases with age. We therefore also calculated relative survival rates for each age group according to stage. Relative survival is an estimation of disease specific survival. It reflects survival of cancer patients, adjusted for survival in the general population with the same age structure. Relative survival is calculated as the ratio of the observed to the expected rates. ${ }^{10}$ Expected survival rates were calculated from life tables for regional male and female populations with the same 5 year age distribution.

\section{RESULTS}

The general characteristics of the patients are shown in table 1. The male:female ratio increased dramatically from 2.1 for patients younger than 60 years to 6.4 for patients aged 70-79 and then decreased to 5.8 for patients aged 80 years or older. The proportion with squamous cell carcinoma was higher among the elderly. The prevalence of concomitant diseases for patients with NSCLC clearly increased with age; in patients aged 70 or older the prevalence of comorbidity was $73 \%$ for men and $61 \%$ for women. The most frequent comorbid conditions in men aged 70 or older were cardiovascular diseases $(31 \%)$ and chronic obstructive pulmonary disease (COPD, 29\%); in older women the most common conditions were cardiovascular diseases (22\%), hypertension $(22 \%)$, and COPD $(20 \%)$.

The proportion of patients with localised NSCLC who underwent surgery with or without radiotherapy was only $9 \%$ of those aged 80 or older compared with $92 \%, 79 \%$, and $61 \%$ in the age groups $<60,60-69$ and $70-79$, respectively. In patients aged 60-69 and 70-79 the proportion who underwent surgery also decreased with comorbidity (fig 1A). In

Table 1 General characteristics of study patients. Data are shown as $n(\%)$

\begin{tabular}{|c|c|c|c|c|}
\hline & Age $<60$ & Age 60-69 & Age 70-79 & Age $80+$ \\
\hline \multicolumn{5}{|l|}{ Sex } \\
\hline Male & $732(68)$ & $1230(84)$ & $1095(86)$ & $230(85)$ \\
\hline Female & $344(32)$ & $233(16)$ & $172(14)$ & 40 (15) \\
\hline \multicolumn{5}{|l|}{ Histology } \\
\hline Squamous cell & $389(36)$ & $725(50)$ & $657(52)$ & $158(58)$ \\
\hline Adenocarcinoma & $388(36)$ & $376(26)$ & $287(23)$ & 40 (15) \\
\hline Large cell undifferentiated & $299(28)$ & $362(25)$ & $323(25)$ & $72(27)$ \\
\hline \multicolumn{5}{|l|}{ Stage } \\
\hline Localised $\dagger$ & $251(23)$ & $403(28)$ & $366(29)$ & $82(30)$ \\
\hline Non-localised & $710(66)$ & $848(58)$ & $694(55)$ & $128(48)$ \\
\hline Unknown & $115(11)$ & $212(14)$ & $207(16)$ & 60 (22) \\
\hline \multicolumn{5}{|l|}{ Comorbidity (n) } \\
\hline 0 & $555(52)$ & $462(32)$ & $303(24)$ & $66(24)$ \\
\hline 1 & $302(28)$ & $506(35)$ & $449(35)$ & 88 (33) \\
\hline $2+$ & $134(12)$ & $397(27)$ & $463(37)$ & $95(35)$ \\
\hline Unknown & $85(8)$ & $98(7)$ & $52(4)$ & $21(8)$ \\
\hline \multicolumn{5}{|l|}{ Comorbidity (type)* } \\
\hline COPD & $159(15)$ & $362(25)$ & $349(28)$ & $72(27)$ \\
\hline Cardiovascular & $122(11)$ & $338(23)$ & $382(30)$ & $80(30)$ \\
\hline Previous cancer & $88(8)$ & 191 (13) & $230(18)$ & 50 (19) \\
\hline Hypertension & $84(8)$ & 209 (14) & $191(15)$ & 29 (11) \\
\hline Diabetes & $47(4)$ & $110(8)$ & $130(10)$ & 31 (11) \\
\hline Other & $93(9)$ & $188(13)$ & $231(18)$ & $44(16)$ \\
\hline
\end{tabular}

*More diseases per patient possible.

†Stage I or II. 

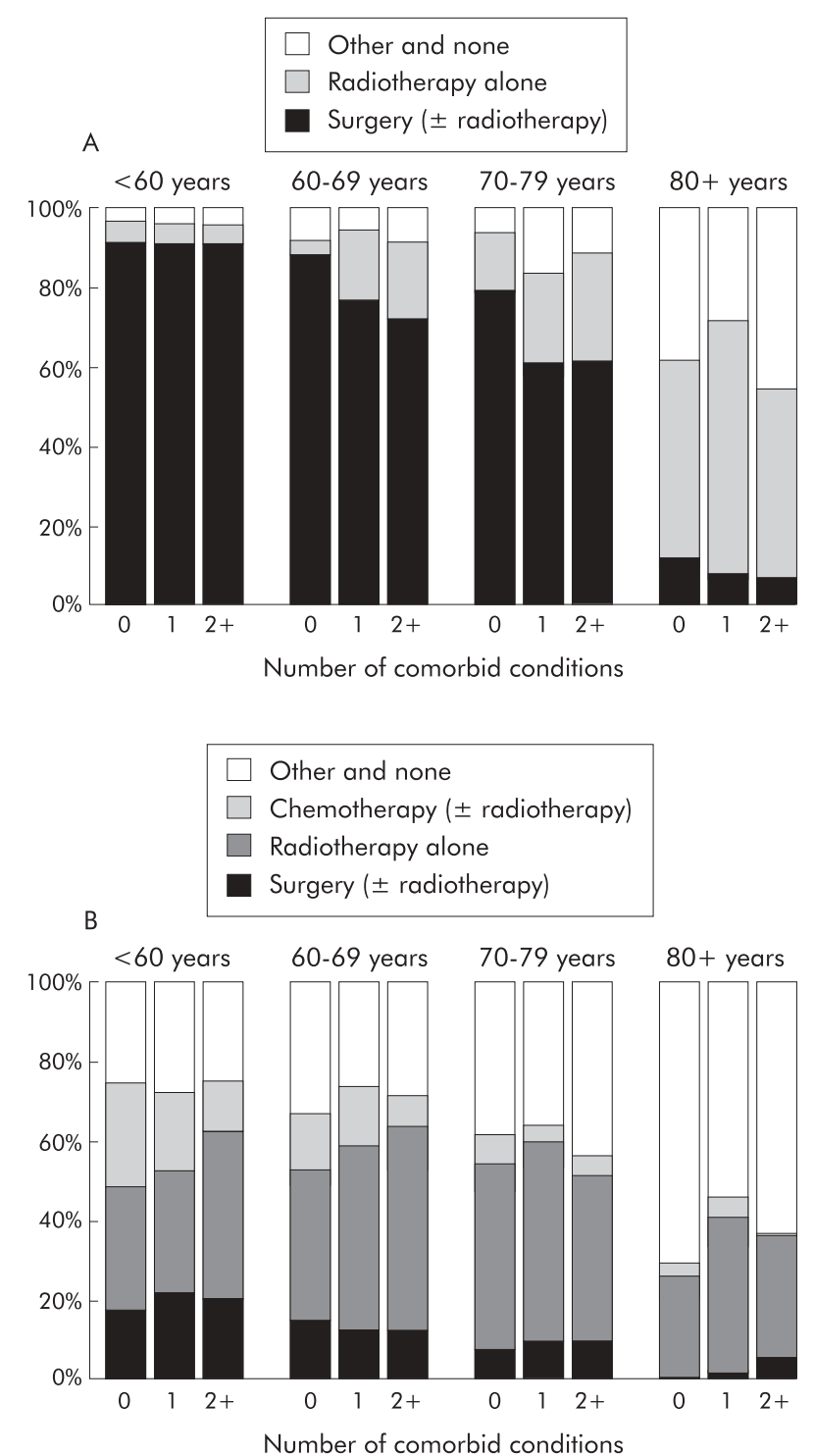

Figure 1 Treatment of (A) localised non-small cell lung cancer (NSCLC) and (B) non-localised NSCLC according to age, comorbidity, and stage.

patients aged 60-69 the proportion who underwent surgery was especially low in the presence of COPD (67\%), COPD and cardiovascular disease $(58 \%)$, or COPD and previous cancer (33\%) compared with $88 \%$ in patients without comorbidity. For patients aged 70-79 the proportion who underwent surgery was $78 \%$ in those without comorbidity but only $59 \%$ in patients with cardiovascular diseases, 55\% of those with previous cancer, $53 \%$ of those with previous cancer in combination with COPD, and $40 \%$ of those with COPD.

Most patients with non-localised NSCLC received radiotherapy alone (fig $1 \mathrm{~B}$ ). The proportion receiving chemotherapy (with or without radiotherapy) was considerably higher in patients younger than 60 years $(24 \%)$ than in those aged 80 or older $(2 \%)$. More elderly patients did not receive any treatment. The number of comorbid conditions had no significant influence on the treatment chosen for patients with non-localised disease. However, for patients with stage IIIa aged 70-79 the presence of COPD lowered the proportion who underwent surgery ( $10 \%$ versus $19 \%$ of patients without comorbidity, results not shown).

Table 2 shows univariate and multivariable analyses of survival for patients with localised NSCLC. Three year survival decreased significantly with age $(\mathrm{p}<0.0001)$ with relative 3 year survival of $62 \%$ for patients younger than 60 and $13 \%$ for those aged 80 or older. The 3 year overall survival decreased from $61 \%$ to $8 \%$. Among patients aged 70 79 the prognosis for those with adenocarcinoma was better than for those with squamous cell carcinoma or large cell undifferentiated carcinoma. Survival was better for Tl tumours and was also better after surgery. The number of comorbid conditions seemed to have no significant influence on survival, but the 3 year survival for patients aged 70-79 was especially low for those with COPD in combination with previous cancer (11\% compared with $38 \%$ for patients without comorbidity). In multivariable analyses age, subtype, tumour size, and the presence of two or more comorbid conditions were independent prognostic factors. The effects of age and tumour size remained significant after treatment was included, but the prognostic effects of subtype and comorbidity disappeared (table 2). In the model with the specific diseases and combinations of diseases only the combination of a previous tumour and COPD had a negative influence on survival. However, this prognostic effect disappeared when treatment was included in the model. This means that the effect of the combination of a previous tumour and COPD might be ascribed to the less aggressive treatment of these patients.

Table 3 shows univariate and multivariable analyses of survival for patients with non-localised NSCLC. One year survival deceased significantly with age $(\mathrm{p}<0.0001)$ with 1 year relative survival of $31 \%$ for patients younger than 60 and $13 \%$ for those aged 80 or older. One year overall survival decreased from $31 \%$ to $11 \%$. Furthermore, histological subtype (only for age group 70-79), stage, and treatment were prognostic factors in univariate analyses. Comorbidity had no influence on survival nor did the specific diseases or combinations of diseases. In a multivariable analysis age and stage were independent prognostic factors. After inclusion of treatment, the prognostic effect of age disappeared and that of stage became weaker but remained significant. In a model with the specific diseases and combinations of diseases, none of the concomitant diseases had an independent effect on survival.

\section{DISCUSSION}

In this population based study we found that the prevalence of serious comorbidity in patients with NSCLC was high, especially in elderly patients and in men. Furthermore, elderly patients were treated less aggressively than younger patients. In patients with localised disease a lower proportion of patients with comorbidity underwent surgery. The survival of patients with localised disease was lower for older patients but the effect of comorbidity on the prognosis was small.

In 2003 the completeness and accuracy of the data on comorbidity were validated in a series of 500 consecutive patients with lung cancer aged 40 and older and diagnosed between 1995 and 1999. Comorbidity scored by the registry team was compared with that scored by a team consisting of a surgeon and an epidemiologist. Recording of comorbidity was correct for about $70 \%$ of patients. There was some underregistration, especially of cardiovascular diseases (internal report). This means that the effects of comorbidity on treatment and survival, as described here, are probably weaker than the real effects.

\section{Prevalence}

The higher prevalence of comorbidity among older patients was expected because the prevalence of diseases generally increases with age. The prevalence of comorbidity in older patients may even be underestimated due to ascertainment bias. Younger patients underwent surgery more often, which 
Table 2 Univariate and multivariable analysis of overall survival for patients with localised* NSCLC according to age, sex, histology, tumour size, comorbidity, and treatment

\begin{tabular}{|c|c|c|c|c|c|c|c|c|c|c|}
\hline & \multicolumn{2}{|l|}{ Age $<60$} & \multicolumn{2}{|l|}{ Age $60-69$} & \multicolumn{2}{|l|}{ Age 70-79 } & \multicolumn{2}{|l|}{ Age $80+$} & \multicolumn{2}{|c|}{ All ages } \\
\hline Total $(\mathrm{n})$ & 251 & & 403 & & 366 & & 82 & & 1102 & \\
\hline Alive at 3 years $(n)$ & 154 & & 198 & & 117 & & 7 & & 476 & \\
\hline \multirow[t]{3}{*}{ Relative 3 year survival (\%) } & $62 \%$ & & $52 \%$ & & $38 \%$ & & $13 \%$ & & $47 \%$ & \\
\hline & \multicolumn{8}{|c|}{ Overall 3 year survival (\%) and univariate $p$ values } & \multicolumn{2}{|c|}{ Multivariable } \\
\hline & $\%$ & p value & $\%$ & p value & $\%$ & p value & $\%$ & p value & HR & p value \\
\hline \multicolumn{11}{|l|}{ Age } \\
\hline$<60$ & 61 & & & & & & & & 0.84 & 0.1 \\
\hline $60-69+$ & & & 49 & & & & & & 1 & \\
\hline $70-79$ & & & & & 32 & & & & 1.28 & 0.007 \\
\hline $80+$ & & & & & & & 8 & & 1.31 & 0.07 \\
\hline \multicolumn{11}{|l|}{ Sex } \\
\hline Female† & 69 & & 51 & & 43 & & 18 & & 1 & \\
\hline Male & 57 & 0.1 & 48 & 0.8 & 30 & 0.1 & 6 & 0.7 & 0.86 & 0.2 \\
\hline \multicolumn{11}{|l|}{ Histology } \\
\hline Squamous cell $\dagger$ & 66 & & 49 & & 30 & & - & & 1 & \\
\hline Adenocarcinoma & 60 & & 53 & & 44 & & - & & 1.10 & 0.4 \\
\hline Large cell undifferentiated & 37 & 0.1 & 38 & 0.5 & 24 & 0.03 & - & & 1.08 & 0.4 \\
\hline \multicolumn{11}{|c|}{ Tumour size } \\
\hline $\mathrm{Tl} \dagger$ & 78 & & 69 & & 42 & & - & & 1 & \\
\hline T2 & 51 & $<0.001$ & 39 & $<0.001$ & 27 & 0.007 & - & & 1.62 & $<0.001$ \\
\hline \multicolumn{11}{|l|}{ Comorbidity } \\
\hline $0 \dagger$ & 53 & & 53 & & 38 & & 10 & & 1 & \\
\hline 1 & 66 & & 48 & & 33 & & 4 & & 0.94 & 0.5 \\
\hline $2+$ & 59 & 0.2 & 43 & 0.2 & 30 & 0.2 & - & 0.5 & 1.13 & 0.2 \\
\hline \multicolumn{11}{|l|}{ Treatment } \\
\hline $\mathrm{RT} \dagger$ & - & & 21 & & 10 & & - & & 1 & \\
\hline Surgery $\pm R T$ & 63 & & 57 & & 47 & & - & & 0.49 & $<0.001$ \\
\hline Other/none & - & & - & $<0.001$ & 2 & $<0.001$ & - & & 1.35 & 0.02 \\
\hline
\end{tabular}

$H R=$ hazard ratio for death; $R T=$ radiotherapy

*Stage I or II.

†Reference category.

Table 3 Univariate and multivariable analysis of overall survival for patients with non-localised* NSCLC according to age, sex, histology, stage, comorbidity, and treatment

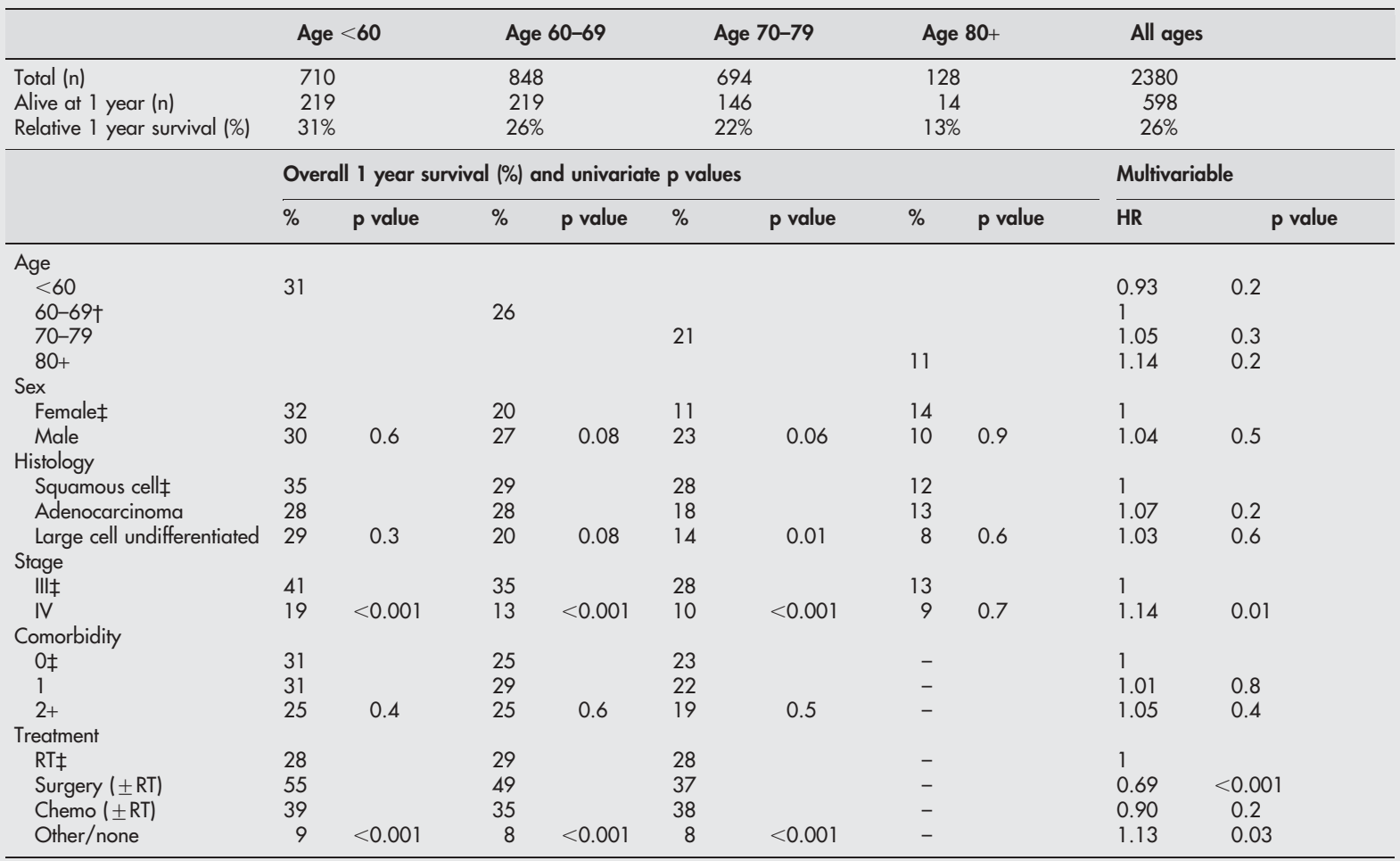

$H R=$ hazard ratio for death; $R T=$ radiotherapy

*Stage III or IV.

†Reference category. 
means that the prevalence of comorbidity reported by the chest physician may be higher in patients with resectable disease because of the required preoperative examination. The high risk of cardiovascular diseases and COPD for patients with lung cancer can be explained by the high proportion of smokers among these patients, especially men.

\section{Treatment}

Patients with localised disease underwent surgery less often when they were older and when comorbidity was present. The resection rate was very low for those with COPD, probably because of the expected higher incidence of postoperative complications and mortality. ${ }^{11}$ However, in everyday practice the resectability is not determined primarily by comorbid conditions but by the effects of comorbidity such as pulmonary and cardiac function.

Age seemed to have more influence on the choice of treatment than comorbidity, especially for patients with localised disease. Apparently, comorbidity alone does not entirely explain why elderly patients with localised disease undergo surgery less often and why those with non-localised disease receive systemic chemotherapy less often. The lower proportion of elderly patients who undergo surgery or chemotherapy was also reported in another area of the Netherlands. ${ }^{12}$ The lower proportion of surgery among elderly patients may be explained by an increased risk of surgical mortality. ${ }^{13-15}$ In previous studies less aggressive treatment of patients with comorbidity has also been shown for breast cancer, prostate cancer, and lymphoma. ${ }^{16-19}$ In contrast, age and comorbidity had a negligible influence on the resection rate in patients with colorectal cancer. ${ }^{20}$ It seems that when surgery is inevitable, as in patients with colorectal cancer, or when no alternative treatment is available, age and comorbidity have a negligible influence on the resection rate. In our study elderly patients received chemotherapy less often than younger patients. In previous studies, however, treatment with vinorelbine was shown to be well tolerated by elderly patients with non-localised NSCLC. ${ }^{21} 22$ The effects of age and comorbidity on the application of chemotherapy for NSCLC in our study may be underestimated because we did not have any information on dose reduction or delay of chemotherapy.

\section{Survival}

Age, stage of disease, and treatment were prognostic factors for patients with NSCLC, independent of sex, histology, and comorbidity. Since age and treatment were both independent prognostic factors for patients with localised disease, the effect of age on the prognosis cannot be completely explained by less aggressive treatment of the elderly.

Overall survival of older patients with lung cancer is worse because of the lower expected survival rate for the elderly in the general population. However, the effect of age remained significant when relative survival rates (adjusted for survival in the general population with the same age structure) were calculated. Since we also adjusted for comorbidity, the lower survival rate for the elderly should be explained by prognostic factors other than comorbidity such as performance status, decreased organ reserves, worse pulmonary function, or psychic and social factors ${ }^{23-27}$ which were not available for analysis in the Eindhoven Cancer Registry.

Comorbidity seemed to have a negligible influence on survival of patients with lung cancer despite less aggressive treatment in case of comorbidity. This contradicts the findings in some other studies where comorbidity was found to be an independent prognostic factor for surgically resected stage I NSCLC, stage III NSCLC, and all lung cancer patients, respectively. ${ }^{26-29}$ However, these studies were not population based and they used other scales for measuring comorbidity (the Kaplan-Feinstein index ${ }^{30}$ and the Cumulative Illness
Rating Scale-Geriatric (CIRS-G) ${ }^{31}$ ). In one of the studies comorbidity influenced overall survival in surgically resected patients with stage I NSCLC in whom comorbidity was rated according to the CIRS-G but not according to the Charlson scale. ${ }^{26}$ In another American study comorbidity count and the Charlson index were significant predictors for lung cancer survival, but explained only $2.5 \%$ and $2.0 \%$, respectively, of the variation in survival. ${ }^{32}$ The influence of comorbidity on survival is probably of less importance in lethal diseases such as lung cancer. Most of these patients die of lung cancer before they have a chance to die of the comorbid condition.

A possible shortcoming of all the studies is the classification of total severity in cases of two or more comorbid conditions. More conditions may have a multiplicative effect rather than an additive effect. Classifying comorbidity as the number of diseases present, or as the sum of scores, or as the most severe condition present may miss the burden of multiple diseases on prognosis. In our data set we also analysed the prognostic effect of the individual diseases and their combinations. However, none of the (combinations of) diseases had an independent effect on prognosis. A negative effect of comorbidity on prognosis may also be cancelled out by earlier detection, with lung cancer possibly being detected at an earlier stage during routine examination for the comorbid condition.

Treatment was a strong prognostic factor, even after adjustment for age. The question therefore arises whether the less aggressive treatment of elderly patients is justified. For careful preoperative selection, studies of the complications during and after treatment should be performed, including data on performance status and pulmonary function.

\section{ACKNOWLEDGEMENTS}

The authors thank the registration team of the Eindhoven Cancer Registry for their dedicated data collection. This work was carried out with grants from the Dutch Cancer Society (IKZ 2000-2260).

\section{Authors' affiliations}

M L G Janssen-Heijnen, V E P P Lemmens, J W W Coebergh, Eindhoven Cancer Registry, Comprehensive Cancer Center South (IKZ), Eindhoven, The Netherlands

S Smulders, F W J M Smeenk, Catharina Hospital, Department of Pulmonary Diseases, Eindhoven, The Netherlands

H J A A van Geffen, Jeroen Bosch Hospital, Department of Surgery, Den Bosch, The Netherlands

J W W Coebergh, Erasmus University Medical Centre, Department of Public Health, Rotterdam, The Netherlands

\section{REFERENCES}

1 Coebergh JWW, Janssen-Heijnen MLG, Louwman WJ, et al, eds. Cancer incidence, care and survival in the South of the Netherlands, 1955-1999: a report of the Eindhoven Cancer Registry with cross border implications. Eindhoven: Comprehensive Cancer Centre South (IKZ), 2001.

2 Janssen-Heijnen ML, Schipper RM, Razenberg PP, et al. Prevalence of comorbidity in lung cancer patients and its relationship with treatment: a population-based study. Lung Cancer 1998;21:105-13.

3 Guadagnoli E, Weitberg A, Mor V, et al. The influence of patient age on the diagnosis and treatment of lung and colorectal cancer. Arch Intern Med 1990;150:1485-90.

4 Monfardini S, Aapro M, Ferrucci L, et al. Cancer treatment in the elderly. Eur J Cancer 1993;29A:2325-30.

5 Wei JY. Cardiovascular comorbidity in the older cancer patient. Semin Oncol 1995;22:9-10.

6 Greenfield S, Aronow HU, Elashoff RM, et al. Flaws in mortality data. The hazards of ignoring comorbid disease. JAMA 1988;260:2253-5.

7 Charlson ME, Pompei P, Ales KL, et al. A new method of classifying prognostic comorbidity in longitudinal studies: development and validation. $J$ Chronic Dis 1987;40:373-83.

8 Mountain CF. A new international staging system for lung cancer. Chest 1986;89:225-33S.

9 World Health Organization. Histological typing of lung tumours. Second edition. Am J Clin Pathol 1982;77:123-36.

10 Hakulinen T, Abeywickrama KH. A computer program package for relative survival analysis. Comput Programs Biomed 1985;19:197-207. 
11 Bolliger CT, Jordan P, Soler M, et al. Exercise capacity as a predictor of postoperative complications in lung resection candidates. Am J Respir Crit Care Med 1995; 151:1472-80.

12 de Rijke JM, Schouten $\amalg$, Schouten HC, et al. Age-specific differences in the diagnostics and treatment of cancer patients aged 50 years and older in the province of Limburg, The Netherlands. Ann Oncol 1996;7:677-85.

13 Finlayson EV, Birkmeyer JD. Operative mortality with elective surgery in olde adults. Eff Clin Pract 2001:4:172-7.

14 Damhuis RA, Schutte PR. Resection rates and postoperative mortality in 7899 patients with lung cancer. Eur Respir J 1996;9:7-10.

15 Alexiou C, Beggs D, Onyeaka P, et al. Pneumonectomy for stage I (T1NO and T2N0) nonsmall cell lung cancer has potent, adverse impact on survival. Ann Thorac Surg 2003;76:1023-8.

16 Satariano WA, Ragland DR. The effect of comorbidity on 3-year survival of women with primary breast cancer. Ann Intern Med 1994; 120:104-10.

17 Lash TL, Thwin SS, Horton NJ, et al. Multiple informants: a new method to assess breast cancer patients' comorbidity. Am J Epidemiol 2003; 157:249-57.

18 Post PN, Kil PJ, Hendrikx AJ, et al. Comorbidity in patients with prostate cancer and its relevance to treatment choice. Br J Urol Int 1999:84:652-6.

19 van Spronsen DJ, Janssen-Heijnen ML, Breed WP, et al. Prevalence of comorbidity and its relationship to treatment among unselected patients with Hodgkin's disease and non-Hodgkin's lymphoma, 1993-1996. Ann Hematol 1999:78:315-9

20 De Marco MF, Janssen-Heijnen ML, van der Heijden LH, et al. Comorbidity and colorectal cancer according to subsite and stage: a population-based study. Eur J Cancer 2000;36:95-9.

21 Gridelli C, Perrone F, Gallo C, et al. Chemotherapy for elderly patients with advanced non-small-cell lung cancer: the Multicenter Italian Lung Cancer in the Elderly Study (MILES) phase III randomized trial. J Natl Cancer Inst 2003;95:362-72
22 Veronesi A, Crivellari D, Magri MD, et al. Vinorelbine treatment of advanced non-small cell lung cancer with special emphasis on elderly patients. Eur J Cancer 1996;32A:1809-11.

23 Eberly LE, Ockene J, Sherwin R, et al. Pulmonary function as a predictor of lung cancer mortality in continuing cigarette smokers and in quitters. Int J Epidemiol 2003;32:592-9.

24 Repetto L, Fratino L, Audisio RA, et al. Comprehensive geriatric assessment adds information to Eastern Cooperative Oncology Group performance status in elderly cancer patients: an Italian Group for Geriatric Oncology Study. J Clin Oncol 2002;20:494-502.

25 Extermann M, Overcash J, Lyman GH, et al. Comorbidity and functional status are independent in older cancer patients. J Clin Oncol 1998; 16:1582-7.

26 Firat S, Bousamra M, Gore E, et al. Comorbidity and KPS are independent prognostic factors in stage I non-small-cell lung cancer. Int J Radiat Oncol Biol Phys 2002;52:1047-57.

27 Firat S, Byhardt RW, Gore E. Comorbidity and Karnofksy performance score are independent prognostic factors in stage III non-small-cell lung cancer: an institutional analysis of patients treated on four RTOG studies. Radiation Therapy Oncology Group. Int J Radiat Oncol Biol Phys 2002;54:357-64.

28 Battafarano RJ, Piccirillo JF, Meyers BF, et al. Impact of comorbidity on survival after surgical resection in patients with stage I non-small cell lung cancer. J Thorac Cardiovasc Surg 2002;123:280-7.

29 Piccirillo JF. Importance of comorbidity in head and neck cancer. Laryngoscope 2000;110:593-602.

30 Kaplan MH, Feinstein AR. The importance of classifying initial co-morbidity in evaluatin the outcome of diabetes mellitus. J Chronic Dis 1974;27:387-404

31 Miller MD, Paradis CF, Houck PR, et al. Rating chronic medical illness burden in geropsychiatric practice and research: application of the Cumulative Illness Rating Scale. Psychiatry Res 1992;41:237-48.

32 Tammemagi CM, Neslund-Dudas C, Simoff M, et al. Impact of comorbidity on lung cancer survival. Int J Cancer 2003;103:792-802.

\section{LUNG ALERT}

\section{Identification of mutations in epidermal growth factor receptor may predict response to gefitinib in NSCLC \\ $\Delta$ Lynch TJ, Bell DW, Sordella R, et al. Activating mutations in the epidermal growth factor receptor underlying responsiveness of non-small-cell lung cancer to gefitinib. N Engl J Med 2004;350: published on-line ahead of print at www.nejm.org (accessed 10 May 2004)}

G efitinib (Iressa) targets the tyrosine kinase epidermal growth factor receptor (EGFR) but the presence of EGFR on tumour cells does not predict response to treatment and in clinical trials only a few patients with non-small cell lung cancer (NSCLC) show any response. In this study the authors sequenced the EGFR gene in NSCLC tumours and found somatic mutations in the tyrosine kinase domain in eight of nine patients who had responded to treatment with gefitinib. Mutations were not found in matched normal tissue from these patients, or in seven patients who had no response to gefitinib $(p<0.001)$. When the most common mutation was transfected into cell lines, enhanced tyrosine kinase signalling was seen in response to EGF compared with the wild type gene and there was an increased sensitivity to in vitro inhibition by gefitinib.

This paper, together with a similar study published recently in Science, raises hope that patients whose tumours will respond to gefitinib could be prospectively identified. On a wider level, it reveals a higher level of complexity in the concept of "targeted therapy": even when key molecular tumour targets have been identified, further work may be required to determine which subgroups will respond.

B S W Choo-Kang

MRC Clinical Research Fellow, Glasgow Royal Infirmary, Glasgow, UK; bsck1p@clinmed.gla.ac.uk 